\title{
Introducing an Annotated Bibliography on Temporal and Evolution Aspects in the Semantic Web
}

\author{
Fabio Grandi \\ DISI, Alma Mater Studiorum - Università di Bologna \\ fabio.grandi@unibo.it
}

\section{INTRODUCTION}

Time is a pervasive dimension of reality as everything evolves as time elapses. Therefore, Web-based information systems and knowledge representation tools at least mirror, and often have to capture, the time-varying and evolutionary nature of the phenomena they model and of the activities they support. This aspect has been acknowledged and long studied in the field of temporal databases [Jensen and Snodgrass 2009] but it truly applies also to the World Wide Web and Semantic Web in particular.

Several papers addressing, in an explicit or implicit way, the representation and management of time and evolution in the Semantic Web appeared recently and, on some aspects, showed a clear upward trend in last years, witnessing a sustained and/or growing research interest. Reflecting and acknowledging such interest, we started in 2011 to collect references concerning the handling of time and evolution issues in Semantic Web research. As it was for [Grandi 2003], the purpose of this collection was to compile a bibliography which could be of help, in particular, to students and young researchers. As a result of such almost endless work, we wrote an annotated bibliography [Grandi 2012], whose latest version is available on the Web at URL:

$$
\text { http://www-db.deis.unibo.it/ fgrandi/ }
$$
TWbib/TSWbib.html

This follows several fortunate bibliographies on timevarying information management, including seven ones on temporal databases [Bolour et al. 1983, McKenzie 1986, Stam and Snodgrass 1988, Kline 1993, Tsotras and Kumar 1996, Wu et al. 1988], two ones on spatio-temporal databases [Al-Taha et al. 1993, Al-Taha et al. 1994], two ones on spatiotemporal data mining [Roddick and Spiliopoulou 1999, Roddick et al. 2000], one on schema evolution [Roddick 1992], one on (temporal) indeterminacy [Dyreson 1996], and one on temporal and evolution aspects in the World Wide Web [Grandi 2003] also advertised on Sigmod Record [Grandi 2004]. Notice that the bibliography we gathered in 2003, already contained the embryo of the present work, with 16 papers dealing with temporal and evolution aspects in the Semantic Web [Grandi 2003, Sec. 2.8].

The collected references, which amount to 768 as of November 2012, are partitioned into two main sections, where they are further organized according to some similarity criterion introduced by brief notes. The former main section (Sec. 2) contains papers explicitly dealing with time or temporal aspects represented in Semantic Web resources or involved in their modelling and management. The latter main section (Sec. 3) contains papers dealing with dynamic aspects of the Semantic Web without explicit reference to any temporal dimension.

We apologize in advance (with the readers and especially with the authors) for any errors, misclassifications and omissions may result from the collected entries. Additions, corrections and comments are obviously welcome. Papers that could have been classified as belonging to more than one section of this bibliography, have actually been assigned to the most representative one, although sometimes such a choice could seem in part arbitrary.

In the following, we introduce the bibliography contents by reproducing here, with the same section organization of the paper, the annotations that have been included. This can be used as a quick reference for locating the section(s) which contain the works of interest in the full bibliography.

\section{TIME AND TEMPORAL ASPECTS}

This first group of collected references is devoted to time and temporal aspects in the Semantic Web. In this collection of 249 papers, we can make a first partition between 137 works properly dealing with modelling and management of temporal Semantic Web resources (in Sec. 2.1 and Sec. 2.2), and 112 works focusing on the study of the semantic and ontological aspects of time itself (in Sec. 2.3 and Sec. 2.4). Within each of the two partitions, we separated papers dealing with time alone (in Sec. 2.1 and Sec. 2.3, respectively) from papers dealing with either time and space (in Sec. 2.2 and Sec. 2.4, respectively). 


\subsection{Temporal Extensions of Semantic Web}

With an approach similar to that employed in temporal database [Jensen and Snodgrass 2009] and temporal XML [Dyreson and Grandi 2009] research, time dimension(s) are explicitly added to Semantic Web languages and formalisms (e.g., RDF, OWL and SPARQL) in order to represent time in semantic annotations, to build temporal ontologies and to support temporal querying and reasoning. The considered time dimension is usually valid time [Jensen et al. 1998], which represents the time when some fact is true in the real world, although other time dimensions have also been considered in some approaches. A number of 97 references has been gathered in this group, where space dimensions have not been considered. Aimed at improving the efficiency of temporal querying and reasoning, optimization techniques investigated in this group involve compact storage solutions and the adoption of ad-hoc index structures for temporal Semantic Web data.

\subsection{Spatio-temporal Extensions of Seman- tic Web}

Among the papers belonging to the temporal extensions group, we can evidence a specific subset of 40 works dealing with time in addition to space dimensions in the Semantic Web. The addition of the space dimension(s) is aimed at supporting spatio-temporal or geospatial knowledge representation and reasoning. Optimized implementation of spatio-temporal query operators has also been considered in a few approaches.

\subsection{Towards an Ontology of Time}

In this subsection, we can found papers concerning the definition of an ontology of time and temporal phenomena. Whereas this problem can sometimes be seen as an application of Semantic Web techniques to the universe of time, it also has a deeper theoretical side which crosses over the boundaries of Semantic Web studies to meet with linguistics and ontological research as philosophical discipline. In this respect, we also included some "classic" studies not belonging to the Semantic Web literature. Neither efficiency nor optimization issues have been considered in this thread, as research interest is definitely focused on semantic aspects.

We start with the listing of 88 collected references, where space and spatial aspects have not been explicitly considered.

\subsection{Towards an Ontology of Time and Space}

Also in the group of works aiming at defining an ontology of time, we can evidence a subgroup of 24 more specific studies concerning the ontological definition of spatio-temporal aspects. References to such works have been collected in this subsection.

\section{EVOLUTION AND VERSIONING AS- PECTS}

In the second main group of collected references we put the studies devoted to dynamic aspects in the realm of Semantic Web without an explicit interest in time and temporal aspects involved in the evolution. In this collection of more than 500 papers, we can make a main partition between papers dealing with evolution aspects (from Sec. 3.1 to Sec. 3.4) and papers dealing with versioning issues (in Sec. 3.5). In this respect, we follow the conceptual distinction between evolution and versioning formalized in the temporal database field [Jensen et al. 1998] for the maintenance of a database schema [Roddick 2009a, Roddick 2009b]. Hence, considering for instance the management of an ontology in the Semantic Web, to support evolution means to permit modifications of the ontology and adaptation of the related resources without requiring maintenance of the previous versions (i.e., the changes are effected by overwriting modified elements and deletions are destructive). On the other hand, supporting versioning means to permit modifications while retaining the previous versions. The maintenance of the whole modification history of the ontology through all its subsequent versions is aimed at continuing the support of legacy applications developed to work with one of the past versions, which is an important requirement in some application fields (e.g., in the legal domain).

The partition of papers dealing with evolution aspects is the most crowded section of the whole bibliography, with a total number of 449 papers. Most of them actually consider evolution of ontologies, even if evolution support for other kinds of Semantic Web resources (e.g., services) has sometimes been considered. In order to highlight the papers dealing with more specific aspects in the context of evolution, we made separate groups of papers specifically dealing with formalization and execution of changes (in Sec. 3.2), design and implementation of editors (in Sec. 3.3) and detection and reasoning about changes (in Sec. 3.4).

\subsection{Evolution Issues}

In this subsection, all the papers generically dealing with evolution of ontologies and Semantic Web resources find place. Although more specific references have been excerpted in subsections from 3.2 to 3.3 , with its 233 entries this is still the more crowded collection, witnessing how the dynamic aspects and evolution problems are outstanding in this field and have received a lot of attention by Semantic Web researchers. Several optimization strategies concerning the engineering process of Semantic Web applications have been also considered, ranging from the coordination of collaborative maintenance efforts to 
the minimization of evolution costs (including costs of applying changes to an ontology and of propagating changes to instances and related resources), from the efficient execution of verification and validation activities to an optimized management of possible inconsistencies.

\subsection{Management of Changes}

As a special subset of the works dealing with evolution in the Semantic Web, we highlight in the collection of 89 references that can be found in this subsection the papers more focused on the management of changes, from the definition and formalization to the implementation of change operations (e.g., ontology updates).

\subsection{Editors for Semantic Web}

Conceptually belonging to the evolution group, we made a separate list in this subsection for the papers describing the design, implementation and usage functionalities of editors for the Semantic Web. Such list consists of a collection of 55 entries gathered in this subsection. In particular, nearly all describe ontology editors.

\subsection{Detection and Analysis of Changes}

Another subset of papers dealing with management of changes in the context of Semantic Web evolution is even more focused on change detection and analysis. In particular, several works are devoted to detection, mining, reasoning and evaluation of ontology changes or of differences between ontology versions. The resulting selection of 72 bibliographic entries can be found in this subsection.

\subsection{Versioning Issues}

This subsection is devoted to the 70 papers most specifically dealing with versioning and management of multiple versions of resources (e.g., of ontologies and RDF graphs, in particular) in the Semantic Web. In such a framework, multi-version settings also include the management of multi-contextual, multidimensional and multi-perspective semantic resources, without an explicit reference to time as a versioning dimension. An optimization often sought in this context is the compact representation of multiversion resources to avoid a storage space growth linear with the number of versions.

\section{AVAILABILITY IN BIBTEX FORMAT}

The whole bibliography is available as a BibTEX file which can be downloaded at URL:

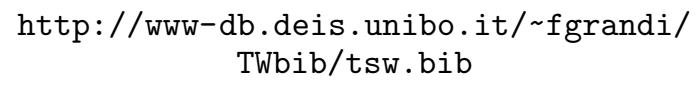

The citation keys for bibliographic entries have been constructed by concatenating the family names of authors and the last two digits of the publication year (plus a lower case letter starting from "a" to disambiguate otherwise equal keys), for papers having up to three authors. When authors are more than three, a form with "-etal" after the family name of the first author has been used in place of the full authors' list. Only the first letter of names is capitalized regardless of the actual presence of capital letters in real names (i.e., a "Pascal case" practice is followed) and compound names have been concatenated and/or simplified (e.g., "van Icks" has become "Vanicks" and "Doe-Moe Woe" has become "Doemoe"); also special characters have been simplified (e.g., "ß乃" becomes "s" and " $\varnothing$ " becomes "o").

\section{ACKNOWLEDGMENTS}

This bibliography could not have been compiled (i.e., in reasonable time) without the support of the World Wide Web and, in particular, without the Google search engine (including the Google Scholar facility), the ACM and Springer Digital Libraries, the IEEE Xplore service, and all the priceless information supplied by the DBLP Computer Science Bibliography with the embedded search engines.

\section{REFERENCES}

[1] Khaled K. Al-Taha, Richard T. Snodgrass and Michael D. Soo. Bibliography on Spatiotemporal Databases. ACM SIGMOD Record 22(1):59-67, 1993.

[2] Khaled K. Al-Taha, Richard T. Snodgrass and Michael D. Soo. Bibliography on Spatiotemporal Databases. Intl' Journal of Geographical Information Systems 8(1):95-103, 1994.

[3] Azad Bolour, Tera Lougenia Anderson, Luc J. Dekeyser and Harry K. T. Wong. The Role of Time in Information Processing: A Survey. ACM SIGMOD Record 12(3):27-50, 1983.

[4] Curtis E. Dyreson. A Bibliography on Uncertainty Management in Information Systems. In Amihai Motro and Philippe Smets (Eds.), Uncertainty Management in Information Systems, pages 415-458, 1996. Kluwer Academic Publishers, Boston, MA.

[5] Curtis E. Dyreson and Fabio Grandi. Temporal XML. In L. Liu and M. T. Özsu (Eds.), Encyclopedia of Database Systems, pages 3032-3035, 2009. Springer-Verlag, Heidelberg, Germany.

[6] Fabio Grandi. An Annotated Bibliography on Temporal and Evolution Aspects in the World Wide Web. Technical Report TR-75, TIMECENTER, http://timecenter.cs.aau.dk/, 2003.

[7] Fabio Grandi. Introducing an Annotated Bibliography on Temporal and Evolution 
Aspects in the World Wide Web. $A C M$ SIGMOD Record, 33(2):84-86, 2004.

[8] Fabio Grandi. An Annotated Bibliography on Temporal and Evolution Aspects in the Semantic Web. Technical Report TR-95, TimeCEnTER, http://timecenter.cs.aau.dk/, 2012.

[9] Christian S. Jensen, Curtis E. Dyreson (Eds.), Michael Böhlen, James Clifford, Ramez Elmasri, Sashi K. Gadia, Fabio Grandi, Pat Hayes, Sushil Jajodia, Wolfgang Käfer, Nick Kline, Nikos Lorentzos, Yannis Mitsopoulos, Angelo Montanari, Daniel Nonen, Elisa Peressi, Barbara Pernici, John F. Roddick, Nandlal L. Sarda, Maria R. Scalas, Arie Segev, Richard T. Snodgrass, Michael D. Soo, Abdullah Tansel, Paolo Tiberio and Gio Wiederhold. The consensus glossary of temporal database concepts - February 1998 version. In O. Etzion, S. Jajodia, and S. Sripada Temporal Databases - Research and Practice, pages 367-405, 1998. LNCS No. 1399, Springer-Verlag, Heidelberg, Germany.

[10] Christian S. Jensen and Richard T.

Snodgrass. Temporal Database. In L. Liu and M. T. Özsu (Eds.), Encyclopedia of Database Systems, pages 2957-2960, 2009. Springer-Verlag, Heidelberg, Germany.

[11] Nick Kline. An Update of the Temporal Database Bibliography. ACM SIGMOD Record 22(4):66-80, 1993.

[12] L. Edwin McKenzie Jr.. Bibliography: Temporal Databases. ACM SIGMOD Record 15(4):40-52, 1986.

[13] John F. Roddick. Schema Evolution in Database Systems - An Annotated Bibliography. ACM SIGMOD Record 21(4):35-40, 1992.

[14] John F. Roddick. Schema Evolution. In L. Liu and M. T. Özsu (Eds.), Encyclopedia of Database Systems, pages 2479-2481, 2009. Springer-Verlag, Heidelberg, Germany.

[15] John F. Roddick. Schema Versioning. In L. Liu and M. T. Özsu (Eds.), Encyclopedia of Database Systems, pages 2499-2502, 2009. Springer-Verlag, Heidelberg, Germany.

[16] John F. Roddick, Kathleen Hornsby and Myra Spiliopoulou. An Updated Bibliography of Temporal, Spatial, and Spatio-temporal Data Mining Research. In Proc. of Intl' Workshop on Temporal, Spatial and Spatio-Temporal Data Mining (TSDM), pages 147-164, Lyon, France, September 2000. LNCS Vol. 2007, Springer-Verlag, Heidelberg, Germany.

[17] John F. Roddick and Myra Spiliopoulou. A Bibliography of Temporal, Spatial and Spatio-Temporal Data Mining Research. ACM SIGKDD Explorations 1(1):34-38, 1999.
[18] Michael D. Soo. Bibliography on Temporal Databases. ACM SIGMOD Record 20(1):14-23, 1991.

[19] Robert B. Stam and Richard T. Snodgrass. A Bibliography on Temporal Databases. IEEE Database Engineering 17(4):53-61, 1988.

[20] Vassilis J. Tsotras and Anil Kumar. Temporal Database Bibliography Update. $A C M$ SIGMOD Record 25(1):41-51, 1996.

[21] Yu Wu, Sushil Jajodia and Xiaoyang Sean Wang. Temporal Database Bibliography Update. In O. Etzion, S. Jajodia and S.M. Sripada (Eds.), Temporal Databases: Research and Practice, pages 338-366, 1988. LNCS Vol. 1399, Springer-Verlag, Heidelberg, Germany. 